\title{
sciendo
}

10.2478/AMB-2019-0016

\section{THE INFLUENCE OF GAIT PATTERN WITH AIDS ON THE PATIENT'S RECOVERY IN AN EARLY PERIOD OF TOTAL KNEE REPLACEMENT}

\author{
D. Vassileva', I. Nedelcheva², S. Mindova', I. Karaganova' \\ 'Department of Public Health and Health Care, Faculty of Public Health and Social Activities, \\ University of Ruse "Angel Kanchev", Bulgaria \\ ${ }^{2}$ Department of Orthopedics and Traumatology, University Hospital "Kanev" - Rousse, Bulgaria
}

\begin{abstract}
Introduction: The total knee replacement is the definitive treatment for knee osteoarthritis (OA). It is an effective intervention to correct OA-related functional limitation. In such patients postoperatively are observed significant differences in the biomechanics of the two knee joints. While bearing the body weight the unoperated knee joint becomes very painful, stiff and incapable of neutral positioning. These factors significantly hamper the initial training in gait with aids in the standard locomotive stereotype immediately after surgery for knee joint replacement, where walking plays an essential role in daily activities and has varied health benefits. Aim: The aim of the study was to check whether different types of gait with aids in the early postoperative period after total knee replacement have a different influence on the recovery process. Material and methods: This study was conducted between January 2017 and July 2018 in the Department of Orthopedics and Traumatology of the University Hospital "Kanev" - Rousse. It included 56 patients with unilateral total knee replacement after gonarthrosis, divided into a control and experimental group, each of 28 patients. All patients in both groups had knee extension deficit of the untreated knee joint. The patients in both groups followed the same physiotherapy programs, performed from about the 2 nd postoperative day to the 21 st day when they were discharged from the department. The difference between the patients in both subgroups was their gait pattern with two axillary crutches. Results and Discussion: There were statistically significant differences between both groups. One of the most significant differences was in ROM. In the experimental group patients, flexion was improved with an average of $20^{\circ}$ more than in the control group patients. Also, in the operated joint, the patients in the experimental group had no contracture, whereas those in the control group showed $15^{\circ}$ at the end of the followup period. Conclusion: The results of the study show that the choice of gait training in the following order "crutches, operated leg, crutches, untreated leg" in patients with extensor deficiency and contracture in the untreated knee is more appropriate than the standard type of training in walking with aids.
\end{abstract}

Key words: total knee replacement, gait patterns with aids, early recovery period, physiotherapy

Corresponding author: Denitza Vassileva, PhD, Section Physiotherapy, Department of Public Health and Social Activity, Faculty of Public Health and Health Care, University of Rousse "Angel Kanchev", 8 Studentska str., Bg-7017 Rousse, e-mail: ddecheva@uni-ruse.bg 


\section{INTRODUCTION}

$\mathrm{T}$ otal knee replacement is the definitive treatment for knee osteoarthritis (OA) and an effective intervention to overcome the OArelated functional limitation [1]. In some cases, the disease is bilateral and affects both knees. Symptomatic OA of the knee affects $10 \%$ of adults over the age of 55 , and a quarter of those affected, are severely disabled [2]. According to the WHO the knee osteoarthritis is the fourth most important cause of disability in women and eight most important causes in men [3]. Gait is adapted by the patient according to pain, deformity or laxity in the joints of the lower extremities [4]. The predominant antalgic gait is often adapted in the patients with knee osteoarthritis [5]. In such patients significant differences in the biomechanics of the two knee joints might be observed postoperatively. The un-operated knee joint bearing the body weight may become very painful as, stiff and unable to assume the neutral position [6]. These factors significantly hamper the initial training in gait with aids in the standard locomotive stereotype immediately after knee replacement surgery, where walking plays an essential role in daily activities and leads to various health benefits [7]. This necessitates the choice of gait with aids suitable for patients with total knee endoprosthesis of the one leg and severe degenerative, structural or functional injuries at the "healthy knee".

\section{AIM OF THE STUDY}

The aim of the study was to check whether different types of gait with aids in the early postoperative period after total knee replacement may have a different influence on the recovery process.

\section{MATERIAL AND METHODS}

This study was conducted between January 2017 and July 2018 in the Department of Orthopedics and Traumatology of the University Hospital "Kanev" Rousse. It included 56 patients with unilateral total knee replacement for gonarthrosis, divided into a control and experimental group, each of 28 patients. The control group consisted of 10 males and $18 \mathrm{fe}-$ males with an average age of 68 years; the experimental group - of 8 males and 20 females with an average age of 69.2 years. The patients in both groups were with knee extension deficit on the unoperative knee joint because of different reasons such as degenerative or traumatic changes in the joint, which leaded to knee extension deficits and lack of "lock in the knee joint". The patients in both groups had the same physiotherapy programs including positional treatment, massage, cryotherapy, continuous passive motions (CPM), active motions, kinesiotape applications, which performed from about the 2nd postoperative day to the 21 st day, when they were discharged from the department. The difference between the patients in both groups was their gait pattern with two axillary crutches. The patients in the control group walked as follows: at the same time putting the two crutches forward, then the operated leg was exported and the untreated leg afterwards. The walking of the experimental group patients was as follows: first putting the crutch carried on the unoperated side, putting the operating leg, then removing the crutch carried from the operating side and placing the untreated leg.

\section{RESULTS}

The following measurements were made during the study and the following indicators were taken into account in both groups: Visual Analogue Scale (VAS), Range of motion (ROM), Western Ontario and McMaster Universities Osteoarthritis Index (WOMAC) and Six - Minute Walk Test (6MWT). Results showed statistically significant differences between patients in both groups.

The mean values of the VAS in the control group at the beginning of the follow-up period were $7.5 \pm 2$ in motion and $5.5 \pm 1.5$ at rest, and in the experimental group $-7 \pm 1.5$ in motion and $6 \pm 0.5$ at rest $(p=0.005)$. A significant difference was observed in the mean values of the final results of VAS in both groups. For the control group at rest, the mean value was $5.5 \pm 1.5$, whereas in the experimental group it was $3 \pm 0.5$. In the case of motion there was also a significant difference $-2.5 \pm 0.5$ in the control and 1 \pm 1 in the experimental group (Fig.1).

The range of motion (ROM) of the knee joints of the two groups was also measured. The average outcomes on the 2 nd postoperative day in the control group were: operated knee: S: $0^{\circ}-20^{\circ}-30^{\circ}( \pm$ $\left.0.5^{\circ} / 10.5^{\circ}\right)$ and untreated knee: S: $0^{\circ}-15^{\circ}-95^{\circ}( \pm$ $1 \% / 5.5^{\circ}$ ). In this period the average outcomes in the experimental group were: operated knee: S: $0^{\circ}-25^{\circ}$ $-30^{\circ}\left( \pm 0.5^{\circ} / 5.5^{\circ}\right)$ and untreated knee: S: $0^{\circ}-20^{\circ}$ $-95^{\circ}\left( \pm 5.5^{\circ} / 10^{\circ}\right)$. On 21 st day after the total knee replacement, the average outcomes of ROM of the knee joints in the two groups were: S: $0^{\circ}-15^{\circ}-60^{\circ}( \pm$ $10^{\circ} / 5.5^{\circ}$ ) for the operated knee; and for the untreated knee: S: $0^{\circ}-15^{\circ}-95^{\circ}\left( \pm 1^{\circ} / 5.5^{\circ}\right)$ in the patients of the control group and S: $0^{\circ}-0^{\circ}-80^{\circ}\left( \pm 0.5^{\circ} / 5.5^{\circ}\right)$ for the operated knee; and of the untreated knee: S: $0^{\circ}-20^{\circ}$ $-95^{\circ}\left( \pm 5.5^{\circ} / 10^{\circ}\right)$ for the patients into experimental 
group. (Fig. 2) The average outcomes for ROM were significant $(p=0,001)$.

A WOMAC score assessment was also done in patients of both groups. In the control group the mean values were: WOMAC Pain Subscale - 17.88 \pm 5.02 decreasing to $12.55 \pm 2.87$ points $(p=0.005)$; WOMAC Stiffness sub-scale $-5.44 \pm 1.33$ and falling to $4.4 \pm 0.4$ points $(p=0.005)$; and WOMAC Function sub-scale - initially $52.48 \pm 10.27$ falling to $25.25 \pm$ 12.38 points $(p=0.005)$. In the experimental group the mean values were: WOMAC Pain Subscale - initially $18.56 \pm 3.22$ decreasing to $7.55 \pm 1.6$ points $(p$ $=0.005) ;$ WOMAC Stiffness sub-scale $-6.62 \pm 0.58$ to $2.3 \pm 0.3$ points $(p=0.005)$; and WOMAC Function sub-scale $-55.37 \pm 8.35$ to $18.05 \pm 8.48$ points $(p=$ 0.005) (Fig. 3).

The Six-Minute Walk Test (6MWT) was conducted only at the end of the follow-up period - on the 21st postoperative day. The reason for the onetime testing is the inability of the patinets to comply to such a complex locomotion process in terms of duration and distance. The walking distance covered by the patients for 6 minutes had mean values of $267.52 \mathrm{~m} \pm 23.68(p=0.001)$ in the control group and $353.64 \mathrm{~m} \pm 35.72$ in the experimental one $(p=$ 0.001) (Fig. 4).
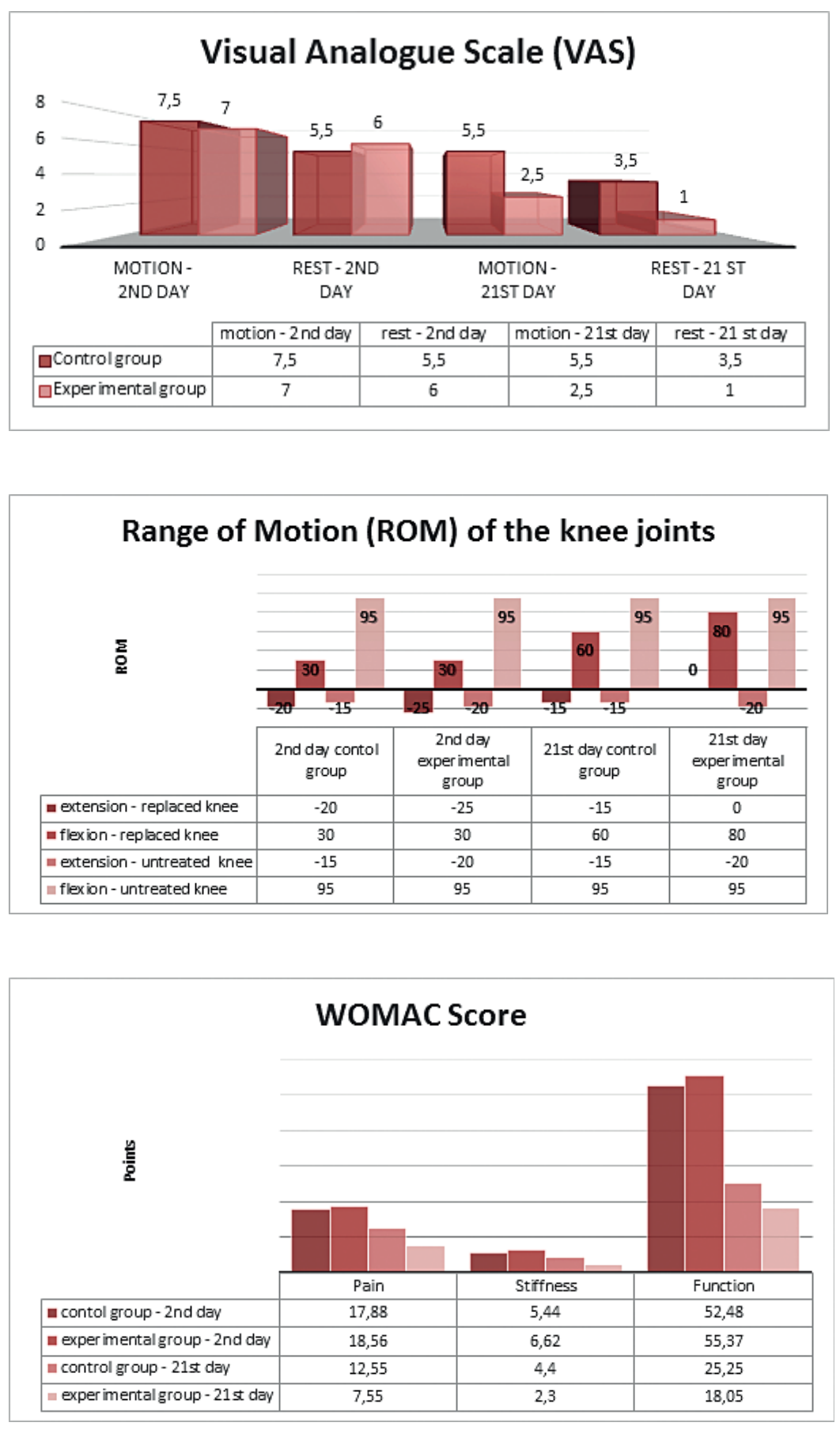

Fig. 1. Changes in mean values of VAS in the both groups

Fig. 2. Changes in the mean values of ROM of the knee joints of both groups

Fig. 3. Changes in the mean values of the WOMAC Score of both groups 


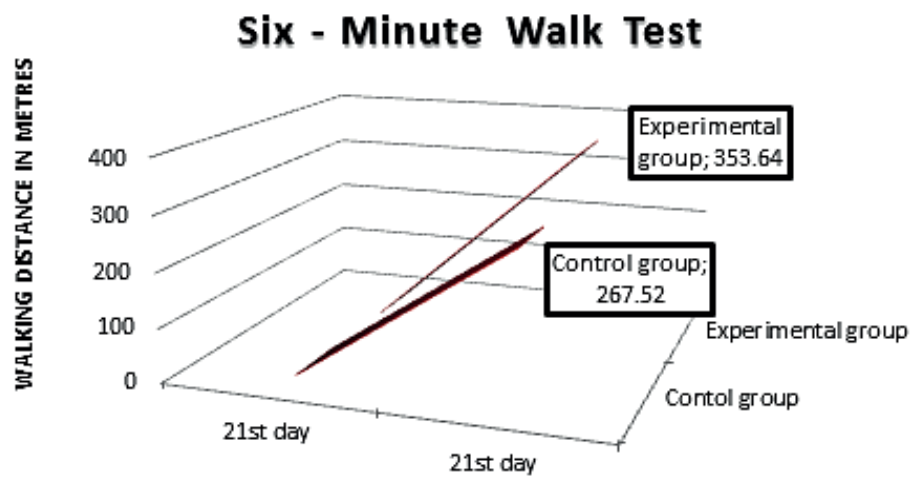

Fig. 4. Changes in mean values of 6MWT of both groups

\section{DISCUSSION}

With similar initial values of VAS at rest and motion in both subgroups, significant differences at study end were found. Thus, in the part of "motion", the difference between the mean initial and final values in the control group is 2 points and it is 4.5 points in the experimental one. In the assessment at "rest", the results of the experimental group were better again, where the difference between the initial and the final mean values was 5 points, whereas the patients in the control group showed an average of 2 . This significant difference of the best results in the endpoints of the two groups could be justified by the better mobility in the replaced joint of the patients in the experimental group which explains the lesser pain during rest and motion.

Examining the mean ROM results of the two groups it becomes obvious that despite similar baseline values in both groups, the final results of those in the experimental group were better. In this patient group, flexion was improved with an average of $20^{\circ}$ more than in the control group. Additionally, patients in the experimental group lacked a contracture in the total knee replaced joint, the, whereas those in the control group had such of $15^{\circ}$ at the end of the follow-up period. This significant difference in the movement of the operated joints in both groups at the end of the approximately 20-day observation period proves that the stereotype of locomotion with aids has a significant impact on this indicator. This means that the properly selected way of walking with aids in accordance with the overall mobility of patients with replaced knee joints may lead to significantly better results in terms of motion range. It can also prevent the occurrence of contractures in the replaced knee due to shortened flexor muscles.
When examining the mean scores from the WOMAC scale, the advantage of the patients in the experimental group becomes clear clearly visible. In the subscale "pain", the mean values of the patients in the experimental group were better, with a difference in the mean end points of 5.68 points in favor of the experimental group. This result can be attributed to better articular movement of the operated joints. In the subscale "stiffness", the result is again in favor of the experimental group with a difference in the final mean values of 3.31 points. This result can also be attributed to better artho-kinematics and a higher range of motion, as well as to the lack of contracture in the operated joint. As a result, the subjective feeling of stiffness in the joint is reduced. In the subscale "function", the experimental group again showed better results with an average of 10.09 points at the final exam. The results from the WOMAC confirm that the choice of stereotype of walking with aids, in particular "crutch, operated leg, crutch, untreated leg", can positively affect not only the individual motion components but also the complex functional activity of the patients with total knee replacement and extensive deficiency of the untreated leg.

The mean values from the 6 MWT also showed a significant difference in the both groups. Patients from the experimental group walked on average $86.12 \mathrm{~m}$ more than those in the control group. Longer locomotion for patients in the experimental group could be explained by less movement pain as well as by better range of motion in the knee joint. This shows that the type of walking with aids (crutches, operated leg, crutch, untreated leg) in patients with contracture in the untreated knee also has a positive impact on the ability to travel over long distances. 


\section{CONCLUSION}

The results of this study showed that the choice of gait training in the following order "crutches, operated leg, crutches, untreated leg" in patients with extensor deficiency and contracture of the untreated knee is more appropriate than the standard type of walking with aids. This type of locomotion provides increased range of motion in the replicated joint, ameliorates the pain syndrome, increases the ability for walking for long distances, and improves functional abilities in performing daily life activities.

\section{REFERENCES}

1. White. DK, Li Z, Zhang Y et al. Physical Function After Total Knee Replacement: An Observational Study Describing Outcomes in a Small Group of Women From China and the
United States; Archives of Physical Medicine and Rehabilitation; 2018,99(1):194-197.

2. Blue C, Coomes S, Yoshida Y. A Novel Downhill Gait - Training Program Following a Total Knee Arthroplasty: A case Report Highlighting the Impacts of Self - selected Speed on Gait Symmetry. J Orthop Sports Phys Ther. 2017; 48, (2):104-110.

3. Baliunas AJ, Hurwitz DE, Ryals AB et al. Increased knee joint loads during walking are present in subjects with knee osteoarthritis; Osteoarthritis and Cartilage; 2002; 10,(7):573-579.

4. Rana P, Joshi S, Bodwal M. Quantitive Gait Analysis in Patients with Knee Osteoarthritis; Int J Physiother Res 2016, 4(5):1648-88.

5. Mündermann A, Dyrby CO, Andriacchi TP. Secondary Gait Changes in Patients With Medial Compartment Knee Osteoarthritis; Arthritis and Rheumatism 2005; 52: 2835-2844.

6. Bejek Z, Paróczai R, Illyés A, Kiss RM. The influence of walking speed on gait parameters in healthy people and in patients with osteoarthritis; Knee Surg Sports Traumatol Arthrosc. 2006, 14: 612-622.

7. Michael JW, Schlüter-Brust KU, Eysel P. The Epidemiology, Etiology, Diagnosis, and Treatment of Osteoarthritis of the Knee; Deutsches Arzteblatt International, 2010; 107(9):152-62. 